\title{
MULTI-FRAME PACKET RESERVATION MULTIPLE ACCESS USING OSCILLATION-SCALED HISTOGRAM-BASED MARKOV MODELLING OF VIDEO CODECS
}

\author{
J. Brecht, M. Del Buono, L. Hanzo \\ Dept. of Electr. and Comp. Sc., Univ. of Southampton, SO17 1BJ, UK. \\ Tel: +44-703-593 125, Fax: +44-703-593045 \\ Email: lh@ecs.soton.ac.uk \\ http://www-mobile.ecs.soton.ac.uk
}

\begin{abstract}
A model is proposed for replacing the video codec in networking studies, which is tested for sources with a mean bitrate in the range of $10 \mathrm{~Kb} / \mathrm{s}$ to $10 \mathrm{Mb} / \mathrm{s}$. The standard frame sizes investigated are the International Telecommunications Union's (ITU) 352 x 288-pixel Common Intermediate Format (CIF), 176 x 144-pixel Quarter CIF (QCIF), 704 x 576pixel 4CIF and $128 \times$ 96-pixel Sub-QCIF (SQCIF). The proposed 20-state 'oscillation-scaled' Markov model was found to represent the relevant video codec characteristics adequately. The performance of this model is then tested in the context of a novel Multi-frame Packet Reservation Multiple Access or MF-PRMA scheme, which is shown in Figure 15 to outperform conventional PRMA in terms of both its delay and packet dropping performance, reaching throughput values as high as $79 \%$.
\end{abstract}

\section{MOTIVATION AND BACKGROUND}

In recent years there have been increased worldwide research activities in the area of modelling video codecs, mainly for studying the performance of multiple access (MAC) schemes [1]-[8], where a simple, but sufficiently accurate video source model is needed. This is motivated by the fact recognised by researchers that in studying the performance of various MAC schemes it is unnecessary and extremely time-consuming to conduct bittrue simulations of the physical layer, including video compression, channel coding and modulation, as long as these physical layer operations can be adequately modelled by an appropriate source model. This is particularly so for example for high-rate, $155 \mathrm{Mbps}$ wireless Asynchronous Transfer Mode (WATM) and other local area networks (LAN), where typically excessive simulation times are encountered in modelling the complex, 1998.

1998 IMAGE COMMUNICATIONS, ELSEVIER, APR.

(C)1998 ELSEVIER. PERSONAL USE OF THIS MATERIAL IS PERMITTED. HOWEVER, PERMISSION TO REPRINT/REPUBLISH THIS MATERIAL FOR ADVERTISING OR PROMOTIONAL PURPOSES OR FOR CREATING NEW COLLECTIVE WORKS FOR RESALE OR REDISTRIBUTION TO SERVERS OR LISTS, OR TO REFUSE ANY COPYRIGHTED COMPONENT OF THIS WORK IN OTHER WORKS MUST BE OBTAINED FROM ELSEVIER.

COMPLETED:14/04/97, REVISED:12/09/97; 12/10/97 high-rate physical-layer simulations. With this motivation Reference [6] provides a review of different models commonly used to mimic the bitgeneration process of voice, data and video sources. In the majority of cases Markov models or their derivatives have been favoured for their simplicity. Other common models are the autoregressive models. A comparison beetween these and a range of other models can be found for example in Reference [3]. Heymann and Lakshman in Reference [7] employed discrete autoregressive (DAR) and Markov models, while Reference [8] has studied the problems associated with the bitrate fluctuation of a video source.

The motivation of this contribution is two-fold. Firstly, a novel video model is proposed and its ability to characterise various video sources is studied in various practical scenarios. The proposed source-modelling technique was investigated in the context of the $\mathrm{H} .263$ video codec $[10,9]$, since this is to date the most successful standard video codec. Although this codec extensively invokes errorsensitive run-length coding techniques for achieving maximum coding efficiency, since it was designed for low errorrate Gaussian channels, in recent years a variety of techniques have been proposed for facilitating its employment in wireless communications. In particular, Khansari, Jalali, Dubois and Mermelstein from INRS in Canada investigated the employment of the H.261 codec, which was the predecessor of the H.263 scheme [11], while Färber, Steinbach and Girod [12] at Erlangen University in Germany as well as Cherriman and Hanzo in the UK [13]-[17] considered various H.263-based video systems. It was found that if the video packets can be superimposed a simple acknowledgement flag in both directions for informing the local and remote encoder of the success or failure of the transmitted packet, the contents of the local and remote reconstruction frame buffers can remain identical, since corrupted packets can be dropped at both ends. This resulted in an extremely robust operation, tolerating packet dropping rates as high as $5 \%{ }^{1}$ The video frame areas represented by the dropped packets can then be updated in future frames. The associated latency or delay of this technique is particularly low in short-frame duration LANs, since the acknowledgement flags become available near-instantaneously.

\footnotetext{
${ }^{1}$ Various H.263 video demonstrations over a range of channel conditions and packet dropping rates can be viewed under http://www-mobile.ecs.soton.ac.uk by means of an MPEG player. Further papers on the corresponding wireless H.263 systems are also downloadable from this address.
} 


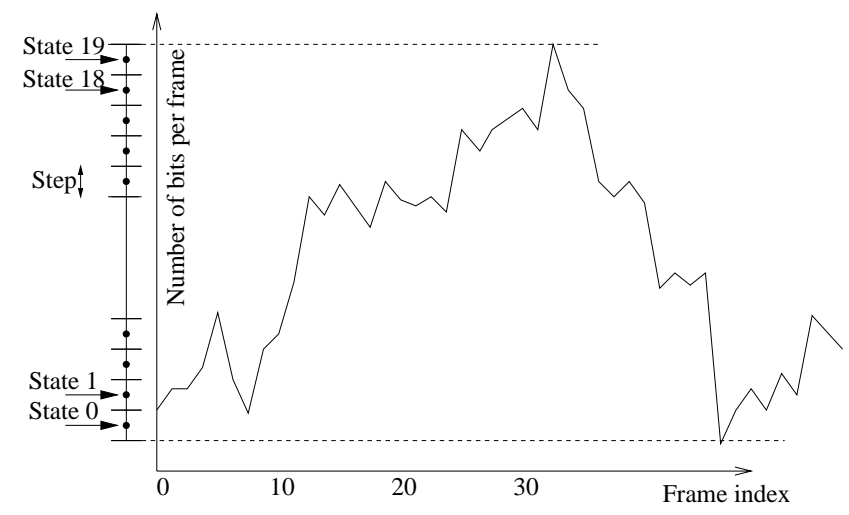

Figure 1: Quantization of the video source-rate fluctuation using a 20 state Markov model.

The second motivation of this contribution is to propose multi-frame packet reservation multiple access (MF-PRMA), as a means of supporting variable-rate multimedia traffic in a wireless local area network (WLAN) scenario. Finally, a number of applications scenarios based on the proposed video models and MF-PRMA are characterised in terms of their throughput and delay performances.

In Section 2 the Markov modelling of video sequences is introduced and the model limitations are highlighted. Section 3 proposes a range of practical improvements to the basic Markov model, while the performance of the algorithm is characterised in Section 4. Our MF-PRMA scheme is outlined in Section 5 and its performance is characterised using the proposed video model in Section 6, leading to our conclusions in Section 7.

\section{MARKOV MODELLING OF VIDEO SOURCES}

In the spirit of our previous discussions we opted for adopting a Markov modulated process, which can adequately model both the first and the second moment of the bitrate fluctuation of various sources. Furthermore, we found that it was possible to superimpose a number of Markov chains in order to account for particular features of the sequence, such as for example spikes in the bitrate histogram and other bitrate irreqularities, as it will be discussed below.

In a Markov Modulated Poisson Process (MMPP) [6] the instantaneous 'arrival rate' of transmitted packets or socalled traffic cells, ie the bitrate generated is 'modulated' by the state of a continuos-time discrete state Markov chain, which will be made explicit during our further discourse. This process is characterised by the arrival rate $\lambda_{i}$ per each state and the mean sojourn time in each state $1 / r_{i}$. The sojourn time has a negative exponential distribution. The arrival rate $\lambda_{i}$ simply corresponds to the mean bitrate in state $i$, while the state transition probabilities are denoted by $P_{i j}$.

In video source modelling the first problem is the choice of the number of states in the Markov-chain. In order to match the bitrate histogram of the original source by that of the model sufficiently accurately, a high number of states is required. However, upon increasing the number of states we found many more bitrate histogram spikes in the simulated sequence than there were in the original. This indicates that

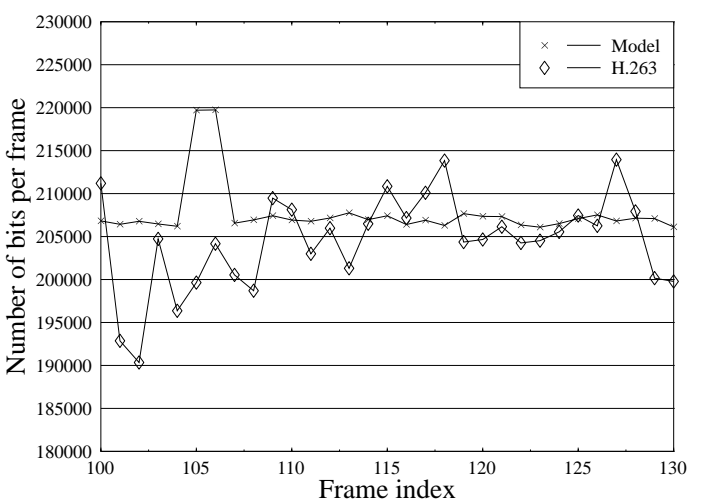

Figure 2: Modelling the H.263-encoded 4CIF Susie sequence at a target bitrate of $7 \mathrm{Mbps}$. For many frames the model resides in the same state resulting in a constant bit rate.

a high number of Markov states requires very long training sequences for generating an accurate state transition matrix in order to arrive at a statistically meaningful number of state transitions amongst all possible states. This issue will be revisited during our further discussions, but suffice to say here that when experimenting with limited-duration practical video sequences, it was impractical to choose a very high number of states, since then the statistical credibility of the investigations became questionable due to the associated low number of state transitions amongst certain low-probability states. We addressed this problem by constructing mosaic-sequences, constituted for example by four different-length sequences combined to form a quadruplesized sequence, which will be invoked in Section 4 for algorithmic performance testing. In order to find a good compromise between these two conflicting requirements after a range of experiments we opted for using a 20-state Markov model for the ITU's H.263 codec.

According to the above considerations we investigated a variety of different resolution sequences to be modelled and after identifying the maximum and minimum bitrate, ie the bitrate range of the sequences, we divided this range in 20 uniform bitrate ranges. At the center of each bitrate interval we allocated a state of the Markov chain, as seen in Figure 1. The transition probability from state $i$ to state $j$ has been found by simulation upon observing the sequence after assigning the actual measured bitrate to one of the 20 states.

Our tentative bit-generation model has the following construction. In each bit-generation cycle a random generator is used to determine the next state of the Markov model, which can be any of the 20 states. These transitions are governed by the transition matrix, generated by evaluating the relative frequencies, approximating the probabilities of all possible Markov-state transitions using simulations. Then in each state the actual number of bits generated obeys the Poisson distribution and the corresponding probability density function (PDF) typically overlaps with those of the adjacent states. Having stipulated the basic video model, let us now scrutinize its behaviour in the next Section. 


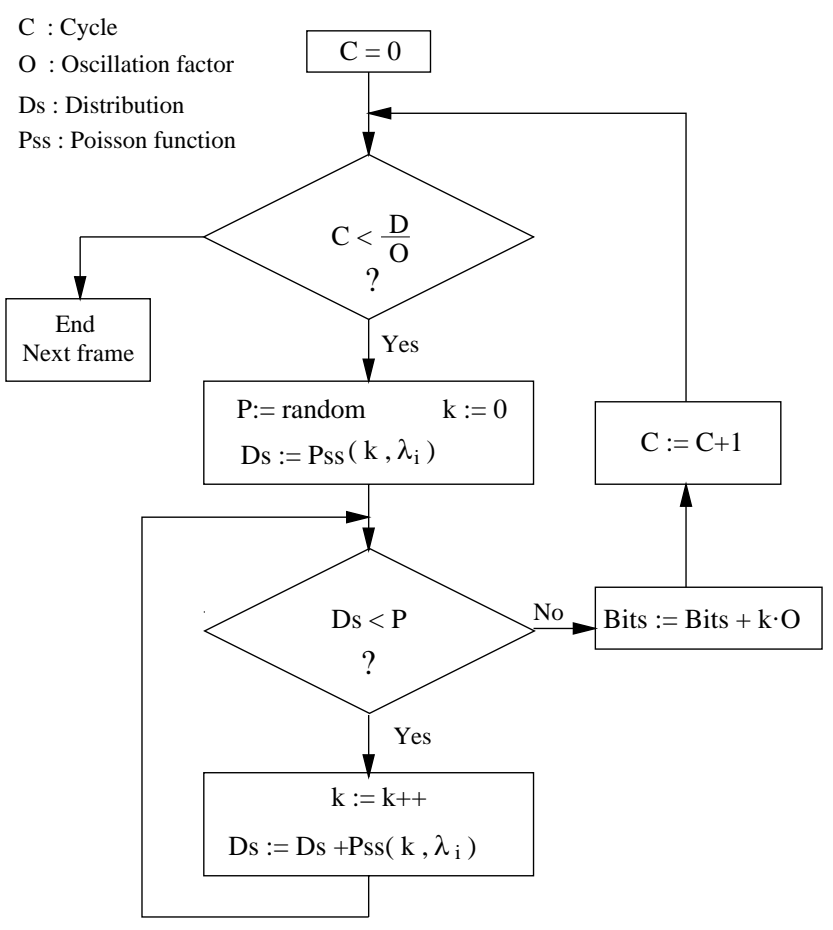

Figure 3: Flow chart of the bit generation algorithm for a video frame

\section{REDUCED-LENGTH POISSON CYCLES}

We note that from a practical point of view operating directly with the number of bits per video frame is inconvenient, since observing the Poisson distribution of

$$
P(n)=\frac{\left(\lambda_{i} T\right)^{n} \exp \left(-\lambda_{i} T\right)}{n !}
$$

we found that the factorial function of the denominator results in an excessive computational demand, when determining the number of bits generated. Hence the solution is to divide the video frame duration into a number of bitgeneration cycles with the advantage that in this way we are able to find the number of bits generated on a more convenient scale, using a granularity more compatible with the typical burst-length of conventional wireless networks. More explicitly, we need to generate the number of bits to be transmitted on a transmission packet-by-packet basis.

Hence we decided to divide the video frame in a number of shorter segments in order to reduce the number of bits generated per Poisson cycle to around 300 or less, simply because in case of a higher average number of bits per generation cycle the probability that we must calculate a factorial higher than 1000! is not negligible. Therefore we opted for invoking a 'division factor' of $D=5000$, which is used to divide the target bit number per video frame in $D=5000$ smaller bit generation cycles. This choice constitutes a good compromise for source rates from $10 \mathrm{Kbps}$ to $10 \mathrm{Mbps}$. For sources at higher bit rates we have to increase the value of $D$.

A consequence is that now the number of bits generated per video frame is the accumulation of the number of bits generated per Poissonian cycle. This means that the distribution is now the convolution of 5000 Poisson distributions. We observed that in this case the number of bits per video

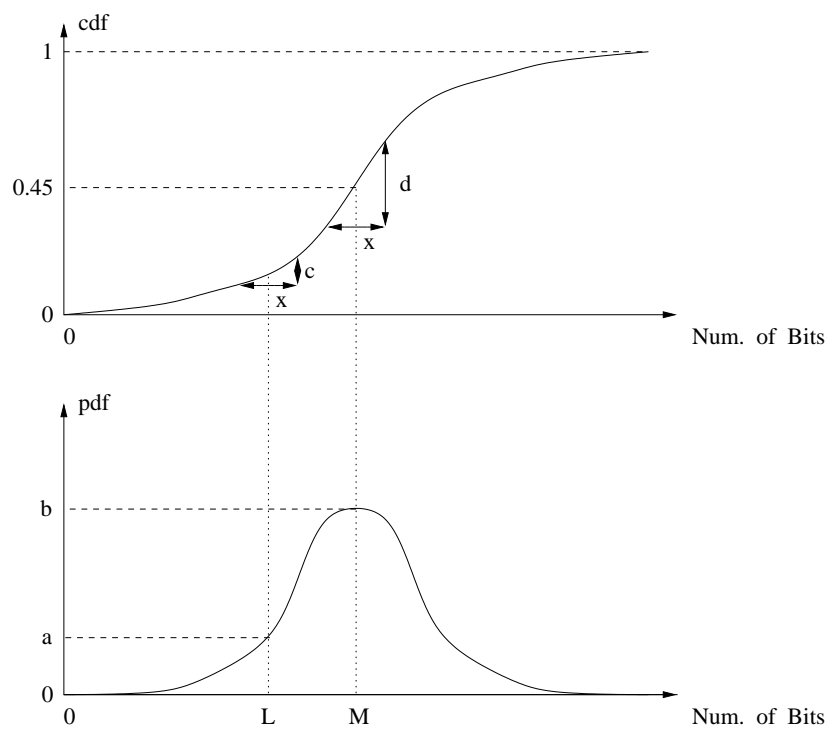

Figure 4: Stylised PDF and CDF

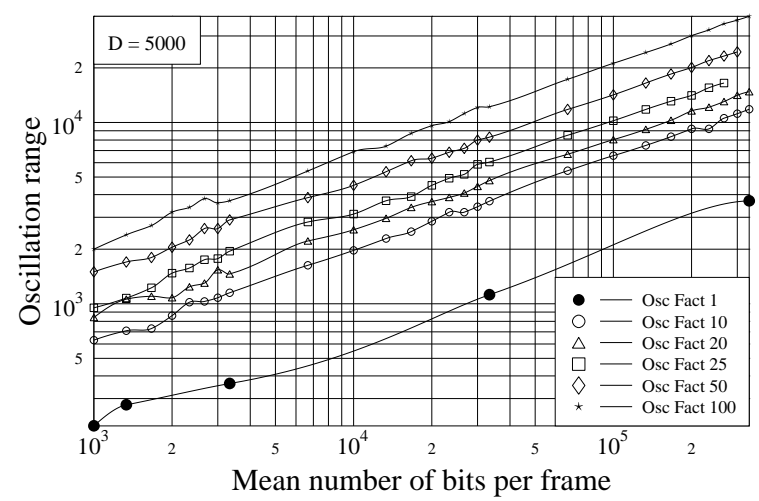

Figure 5: Oscillation range $\Delta R$ versus mean number of bits per frame $R_{a}$ : different ranges can be selected using different $O$ values in order to ensure a better fit of the bitrate histograms of the model to that of the codec modelled.

frame was not sufficiently spread around the average in order to provide a statistically sound model of the bitrate fluctuation for the H.263 codec. This is demonstrated in Figure 2 , in comparison to the actual number of bits generated by the H.263 codec for the high-resolution $704 \times 576$ pixel $4 \mathrm{xCIF}$ (Common Intermediate Format) 'Susie' sequence coded with a target bitrate of $7 \mathrm{Mbps}$. Observe that apart from a single excursion to a state corresponding to about 220 000 bits per frame, which occurs at frame index 105, the process resides in a state emitting a Poissonian distributed rate around 207000 bits per frame. However, these rate fluctuations appear quite limited.

In order to avoid this near-constant bitrate problem we introduce an 'oscillation factor' $O$, the role of which and the terminology becomes explicit below. The effective number of bit generation cycles is now computed as $D / O$ but at the end of each modelling cycle we multiply the number of bits generated, namely $k$, by the value of $O$. This measure al- 


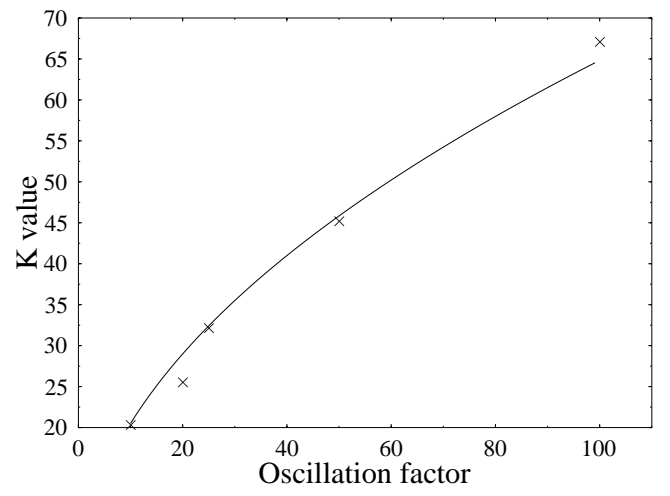

Figure 6: The value of $\mathrm{K}$ for different oscillation factors $O$, where the curve was found by minimum mean-squared fitting

lows us to maintain a better bitrate granularity as it will be demonstrated below. The operation of the model is illustrated in the flow-chart of Figure 3, which will be described with reference to the relationship between the Poissonian $\mathrm{PDF}$ and CDF in Figure 4.

For the sake of augmenting the inner working of the flow-chart in Figure 3 let us consider the stylised Poissonian CDF and PDF of Figure 4 and initially let us assume an oscillation factor of $O=1$. There are thus $D / O=5000$ Poissonian generation cycles required in order to generate a whole video frame, ie the main loop of the flow chart, in which the index $C$ is incremented, is passed through 5000 times. At the commencement of each bit generation cycle, a uniformly distributed random number is generated and assigned to $P$, where $0<P<1$. This corresponds to choosing a random value on the vertical axis of the CDF of Figure 4, which is 0.45 in our example. Then the Poisson PDF of Equation 1 is evaluated for the iteration index of $k=0$ and the returned Poissonian value $\operatorname{Pss}\left(k, \lambda_{i}\right)$ of Equation 1 is assigned to the variable $D s$. A Poissonian generation of bits is achieved when the values of the Poisson PDF, Pss $\left(k, \lambda_{i}\right)$, are accumulated in the variable $D s$ for incremented values of $k$, until $D s \geq P$, which is true above $k=M$ in our example. The index $k=M$, multiplied by the oscillation factor $O$, then represents the number of bits generated in the current cycle. This number is then added to the contents of the counter 'Bits' in the flowchart, where 'Bits' was initialised to zero at the commencement of each new generation cycle. In order to augment our exposition further, here we note that the bitrate granularity or resolution is one bit without using our proposed oscillation factor, but for non-unity oscillation factors it becomes identical to the factor itself. This is attractive, since in high-rate scenarios, where $O$ is high, a higher absolut bitrate granularity is maintained.

The example of Figure 4 justifies that the numbers of bits per cycle generated as described above is Poissoniandistributed. In general, the distribution is Poissonian, if the probability of generating $M$ bits is a factor $b / a$ higher than the probability of generating $L$ bits, where $M, L>0$, while $a$ and $b$ are the Poisson PDF values for abscissa values of $L$, $M$, respectively. Note that these abscissa values are discrete and hence the Poisson PDF is a discrete histogram. Upon taking into account that the PDF represents the gradient of the CDF, we have $b / a=d / c$ in Figure 4 . If we now consider that for geometric reasons, the probability of coining a uniformly distributed $P$ value in the wider interval $d$, ie generating $M$ bits, is a factor $d / c$-times higher than that of randomly picking one from the shorter interval $c$, which corresponds to generating $L$ bits, the Poissonian nature of the generation is proved.
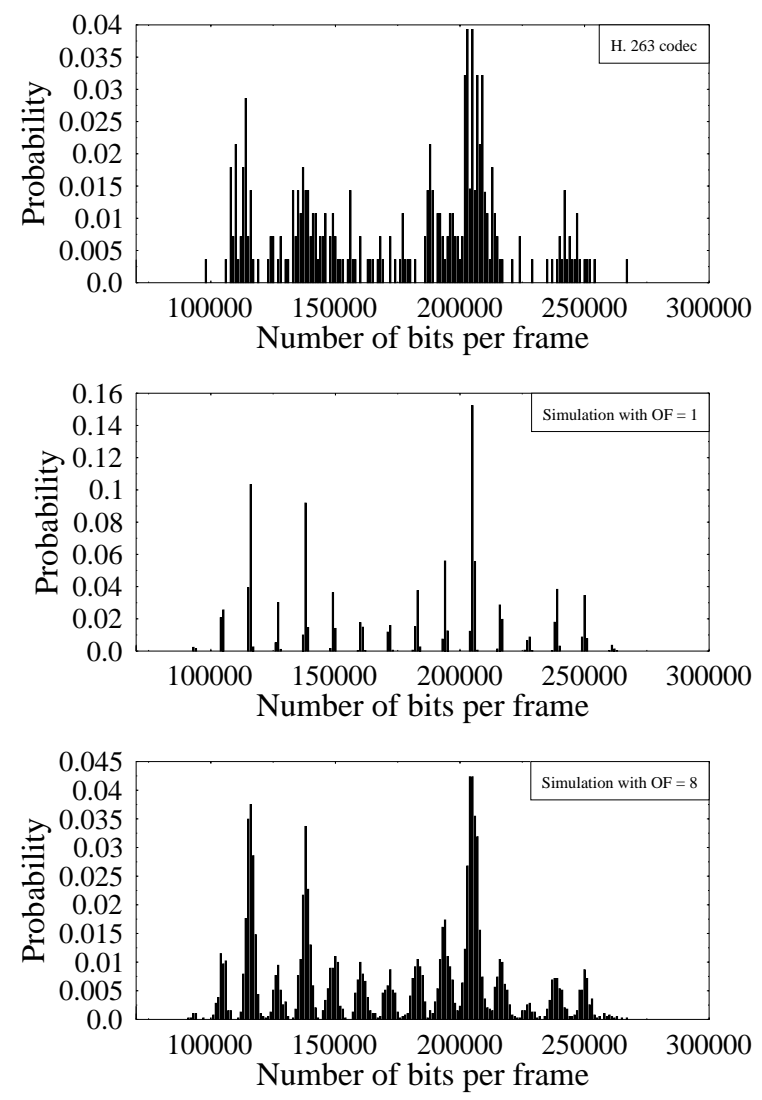

Figure 7: Bitrate histograms for the 4CIF Susie sequence at a $5 \mathrm{Mbps}$ target bitrate generated by the $\mathrm{H} .263$ codec and by the proposed model with $O=1$ and $O=8$. Observe the better histogram fit due to a higher value of the $O$.

In order to study the effect of different oscillation factors $O$ the following experiment was carried out. Initially, $O=1$ was stipulated, resulting in $D=5000$ Poissonian cycles per videoframe, yielding 5000 numbers, each generated by a Poisson process using Equation 1, where $\lambda_{i}$ is the mean bitrate of the Markov chain in state $i$ and $T=$ Frame Duration $/ D$. These simulations were conducted for 3000 video frames. The lowest and the highest number of bits generated were recorded and we refer to their difference as the 'bitrate fluctuation range'. This range was then recorded for various average bitrates or mean number of bits per frame. The results are plotted in Figure 5 for a range of $O$ values between 1 and 100 .

Explicitly, the curve plotted for $O=1$ is in fact the original curve, where we used $D=5000$ Poisson cycles per video frame. It is easy to observe in the Figure that for example for an average source rate around $5 \mathrm{Mbps}$, corresponding to 
167000 bits per video frame at 30fps, a fluctuation of 3000 bits around the mean value of 167000 bits is almost negligible. This phenomenon was observed earlier in Figure 2.
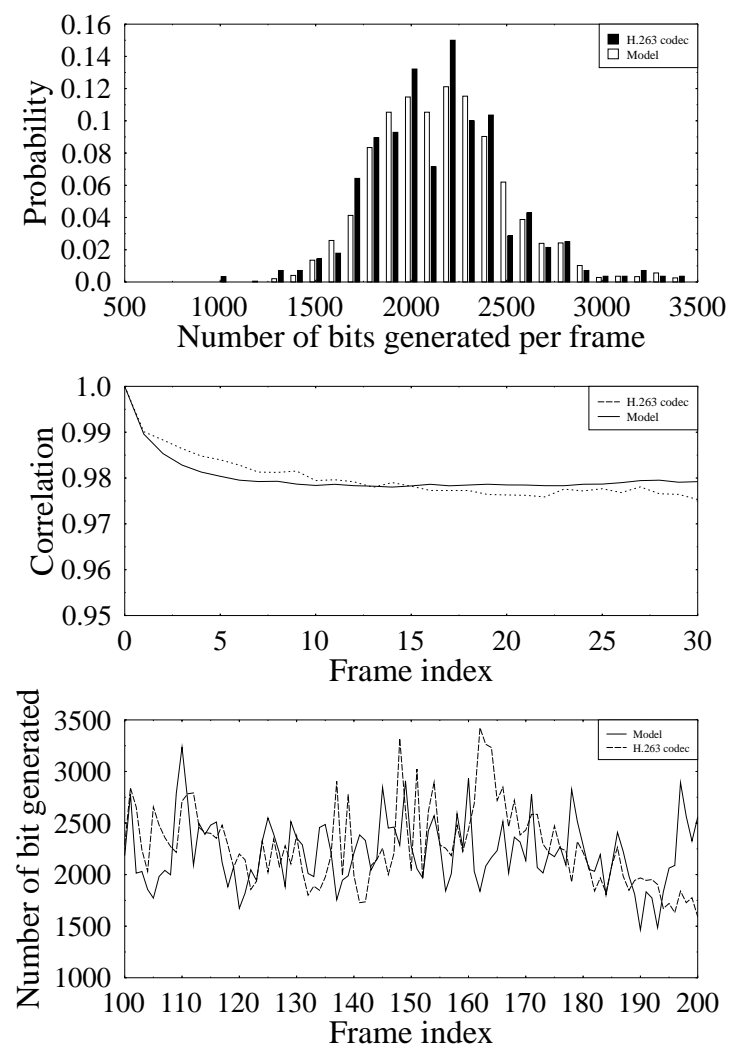

Figure 8: Bitrate histogram, correlation and typical bit rate for the Carphone sequence. Comparison between the H.263 codec at QCIF size, 64kbps target bitrate and the Markov model

Observe furthermore in Figure 5 that due to the introduction of an oscillation factor of $O=100$ for a $5 \mathrm{Mbps}$, 30 frames/s, 167000 bits per frame scenario, the fluctuation range now becomes significantly higher, approximately 30000 bits around the mean value. This is because for $D=5000$ and $O=1$ the number of bits per frame was the cumulative value of 5000 Poissonian variables, yielding a near constant value. By contrast, for $O=100$ the higher oscillation range is a consequence of accumulating only 50 such variables.

At this stage a further step was required in order to complete the model design. Specifically, given an overall average bit rate of $R_{a}$, a minimum and maximum bitrate of $R_{\min }$ and $R_{\max }$, respectively, as well as a set of $N$ Markovmodel states, the resulting target bitrate $R_{i}$ ( or $\lambda_{i}$ ) of state $i$ with $0 \leq i \leq N$ is given by:

$$
R_{i}=R_{\min }+i \frac{R_{\max }-R_{\min }}{N}=R_{\min }+i \Delta R .
$$

While residing in any of the Markov-states, the model will ensure that the range of the instantaneous bitrate-fluctuations is limited to $\Delta R=\left[R_{\min } ; R_{\max }\right]$, and the specific
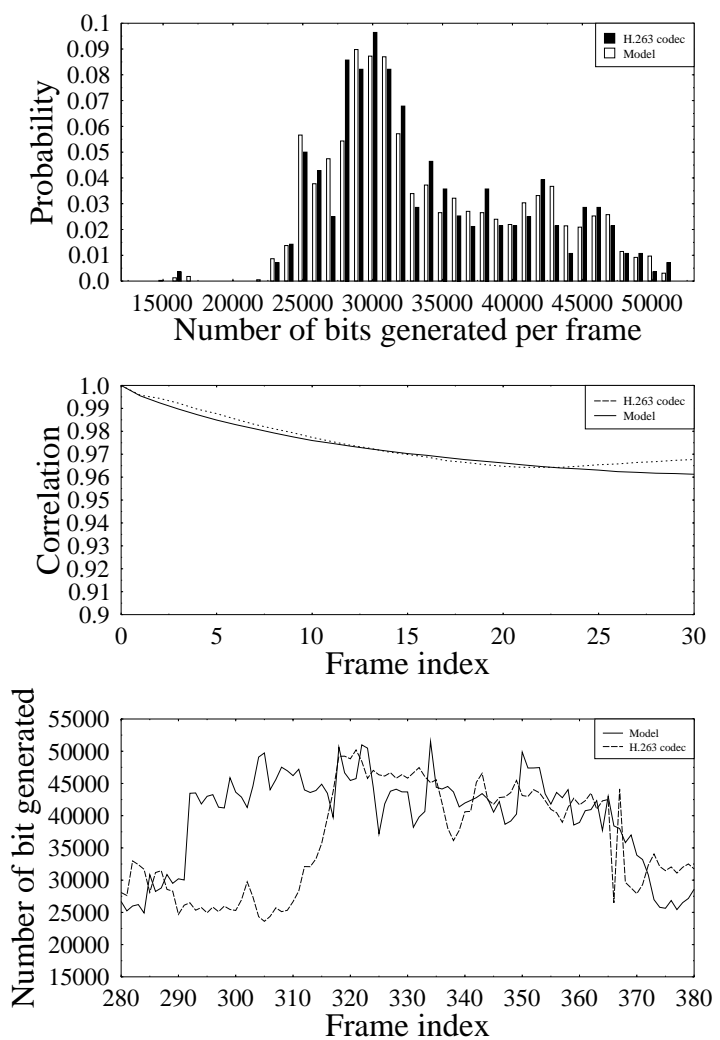

Figure 9: Bitrate histogram, correlation and typical bitrate for the Susie sequence. Comparison between the H.263 codec at $4 \mathrm{CIF}$ size, $1 \mathrm{Mbps}$ target bitrate and the Markov model

bitrate values of each state associated with a certain mean bitrate obey the Poissonian distribution. Given the oscillation range $\Delta R$, we can invoke Figure 5 in order to determine the required oscillation factor $O$.

In order to assist in this, we found an empirical relation between the quantities involved. From Figure 5, which is plotted on a log-log axis, we inferred that the relationship between the average value of bits per frame $R_{a}$ and the oscillation range $\Delta R$ is given by:

$$
\Delta R=K \sqrt{R_{a}} .
$$

Minimum mean-squared fitting of the experimental $R_{a}$ and $\Delta R$ values for various $K$ values revealed the following dependence of $K$ on the oscillation factor $O$ :

$$
K=a \cdot \sqrt{O}
$$

where we have $a=6.48$, yielding

$$
\Delta R=6.48 \cdot \sqrt{O \cdot R_{a}} .
$$

The goodness-of-fit of this matching process is characterised in informal terms by Figure 6 .

The above mentioned experimental relationship has been used in our simulations and the corresponding bitrate histograms are depicted in Figure 7 for two different $O$ factors, 

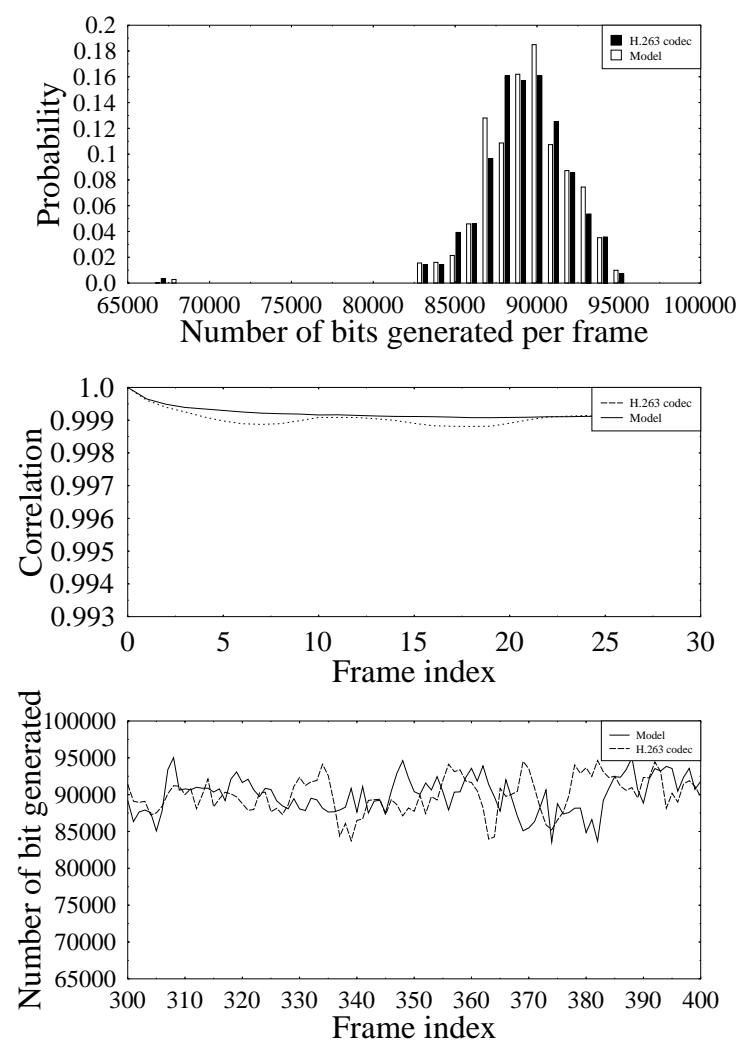

Figure 10: Bitrate histogram, correlation and typical bitrate for the Miss America sequence. Comparison between the H.263 codec at CIF size, 3Mbps target bitrate and the Markov model

namely for $O=1$ and 8 , as well as for our experimental data generated by the H.263 codec for the 4CIF 'Susie' sequence, while maintaining an average bitrate of $5 \mathrm{Mbps}$. Observe in the Sub-Figure in the middle that in accordance with our previous experience, for $O=1$ there is only a very limited bitrate fluctuation or spread within the Markov-states around the target bitrates of the individual Markov states. However, for $O=8$ a more appropriataly spread Poissonian bitrate distribution is observed in each state, where $\Delta R$ is a factor $\sqrt{8}$ higher. Observe for $O=8$ at the bottom of the Figure that the PDFs are slightly more spread towards the top end of the bitrate range than in the lower-rate Markov states. This is because for the Poisson distribution the value of the variance is equal to the mean value, which is clearly higher for the the states closer to the top end of the bitrate scale.

\section{VIDEO MODEL MATCHING}

From our simulation results we found that for a source bitrate around $1 \mathrm{Mb} / \mathrm{s}$ or less an oscillation factor between $O=1$ and 3 was appropriate. For source rates around $10 \mathrm{Mb} / \mathrm{s}$ a value around $O=50$ was required, depending on the target source rate. Furthermore, we found that the model was quite flexible and allowed us to emulate a range of different video scenes adequately, always obeying Equa-
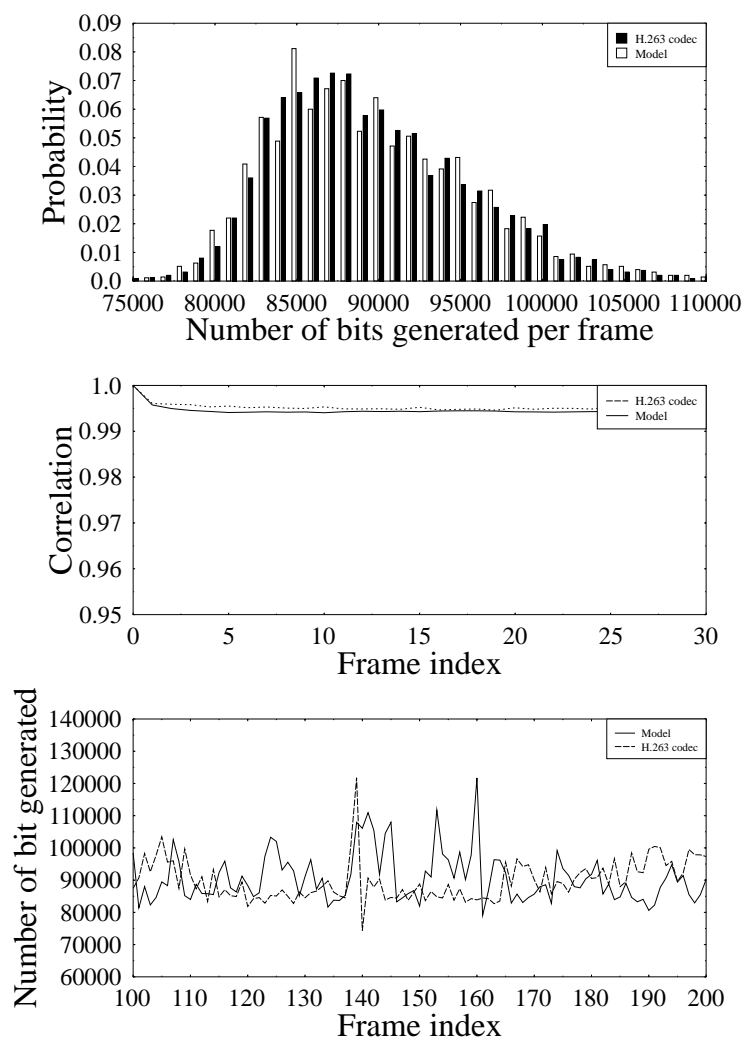

Figure 11: Bitrate histogram, correlation and typical bitrate for the mosaic sequence. Comparison between the H.263 codec at CIF size, 2.6 Mbps target bitrate and the 30-state Markov model

tions 3-5.

Figures 8-11 show a number of model characteristics for various video sequences. Specifically, at the bottom of each of these Figures the typical bitrate fluctuation of the original H.263 codec and that of the model can be seen, as an easily interpreted illustrative example. The frame indeces chosen represent arbitrary typical segments of four different video clips at different resolutions. In the centre of each of these illustrations the normalised correlation between the bitrates of consecutive frames was plotted, while at the top the bitrate histogram of both the original experimental data and that of the model is displayed. Assuming that the original H.263 bitrate is denoted by $x(n)$ and the model's rate by $y(n)$, the bitrate correlation was defined as:

$$
r_{x y}=\frac{\sum_{0}^{30} x(n) \cdot y(n)}{\sum_{0}^{30} x^{2}(n)} .
$$

A representative range of low, medium and high bitrate scenarios at $64 \mathrm{kbps}, 1 \mathrm{Mbps}$ and $3 \mathrm{Mbps}$, respectively, were studied using various video sequences encoded at various bitrates. Although the bitrate histograms would not be acceptable at high confidence-level using rigorous goodness-offit distribution testing techniques, such as the KolmogorovSmirnov- or the $\chi^{2}$-test [18], for practical network modelling purposes they were deemed informally adequate. The 
bitrate correlation functions also exhibited an adequate informally judged match. The observed deviations from experimental features were deemed to be a consequence of the limited-duration training data for the model, which adopted the transition matrix probabilities found from our experimental video data. When using these state-transition probabilities, extremely long training sequences and model verification experiments would be required for achieving a better statistical match. Figure 10 shows a very high correlation due to the particular features of the 'Miss America' sequence, which exhibits a rather limited motion activity and hence the number of bits generated per frame is almost constant without large excursions around the mean value.

In order to overcome the problem of having short training sequences we attempted to create longer sequences. Four QCIF sequences have been combined to obtain a quadruplesized CIF sequence. Since each QCIF component sequence has a different length in terms of the number of picture frames, it is possible to create an arbitrarily long CIF sequence by playing the constituent sequences back and forth. These experiments have been carried out for 3000 video frames using the sequences 'Sales-man, Carphone, Newscaster and Foreman' in order to mix high-dynamic and low-dynamic sequences. The results are shown in Figure 11. A 30-state Markov model has been used because the high number of frames available allowed us to find a sound set of transition matrix statistics. Having characterised the proposed video model, let us now introduce the concept of our multiple access scheme, namely MF-PRMA.

\section{MULTIPLE ACCESS}

An excellent overview of various wireless multiple access schemes was provided by Li and Qiu in Reference [23]. A relative of slotted ALOHA (S-ALOHA), Packet Reservation Multiple Access (PRMA) was proposed by Goodman et al $[19,20]$ for wireless networks, where the base station (BS) informs the portable stations (PS) as to the status of the slots in each PRMA frame in its broadcast message. Unused slots are contended for by the PSs, but since they are unaware of eachother's contentions, collisions may occur in case of simultaneous transmission of more than one packets in the same slot. In this case none of the contending PSs acquire the slot for future transmissions. Consistent future collisions are prevented by introducing a so-called permission probability that allows terminals to re-contend according to this system parameter, which is optimised as a function of the number of slots to content for. This optimisation process will be demonstrated in the next Section. By contrast, in case of uncontended transmission the PS reserves the slot in each PRMA frame, as long as it has packets to transmit. In case of high traffic loads many collisions occur, leading to wasting a large fraction of the slots and hence degrading the system's throughput. The PRMA protocol was further developed by Dunlop et al [21] under the name of PRMA++ by allowing contention in a fraction of the slots, which protected the information slots from collisions.

Since tele-traffic is typically time-variant, it is desirable to assign different amounts of resources according to the sources' behaviour. Dynamic Time Division Multiple Access (D-TDMA), was proposed for integrated voice and data communication [23], where so-called 'request minislots' are accessed like in S-ALOHA and the 'information slots' are assigned by a centralised method. Another efficient resource assignment technique proposed by Amitay and Nanda is Resource Auction Multiple Access (RAMA) [22], where only one user is granted access to the system at any instant, hence preventing collisions. Lastly, Dynamic Reservation Multiple Access (DRMA) was suggested by Li and Qiu [24].

A possible approach to supporting multi-rate users in Packet Reservation Multiple Access (PRMA) is the introduction of a hierarchical structure of PRMA multiframes, which comprise a certain number of frames. In this treatise we refer to this protocol as Multiframe PRMA (MFPRMA). In conventional Time Division Multiple Access (TDMA) systems a terminal can only reserve one slot per frame. In MF-PRMA, however, if $M$ is the number of frames per multiframe, a reservation of $n_{R}<M$ slots per multiframe is possible and corresponds to a 'reservation rate' lower than that of one slot per frame. The term reservation rate is introduced in order to describe the rate of a temporary virtual channel occupied by one terminal holding a reservation. The reservation rate can be reduced down to $(1 / M)^{t h}$ of the reservation rate of the original structure. Initially, we assume that a certain reservation rate is associated to each column of the multiframe structure of Figure 12. This will be referred to as the 'reservation level' of the column. Figure 12 displays an example for two columns, where a high-rate video user would choose the column with a high reservation rate, one slot per frame, while a speech user or a low-rate video user only needs one slot every fourth frame and is transmitting in slots of the appropriate column. A terminal seeking to transmit packets for a certain service will thus contend in a column with the reservation level that is most suitable for the required service. The introduction of a reservation expiry time, which allows a delayed reservation cancellation, reduces the number of contentions and improves the performance of a MFPRMA system. Let us now evaluate the performance of such a scheme in the next Section.

\begin{tabular}{lccc}
\hline Definition & Notation & Unit & Value \\
\hline Channel Rate & $R_{C}$ & Mbps & 50.0 \\
Source Target Rate & $R_{S}$ & Mbps & 1.0 \\
Gross Slot Size & $S_{G}$ & bit & 1024 \\
Net Slot Size & $S_{N}$ & bit & 424 \\
Slots per Frame & $N$ & & 64 \\
Slots per Uplink Partition & $N_{U}$ & & 29 \\
Maximum Delay & $D_{m a x}$ & sec. & 0.03 \\
Max. Number of Reserved & & & \\
Slots/Frame/Terminal & $n_{R}$ & & 10 \\
Frames per Multiframe & $M$ & & 16 \\
Reservation Expiry Time & $T_{e x p}$ & slots & 0 \\
Permission Probability & $p$ & & $0.1 . .0 .4$ \\
Number of Video Terminals & $\mathrm{U}$ & & 7
\end{tabular}

Table 1: MF-PRMA Parameters for a 1 Mbps video service 
SLOTS PER TDD - FRAME

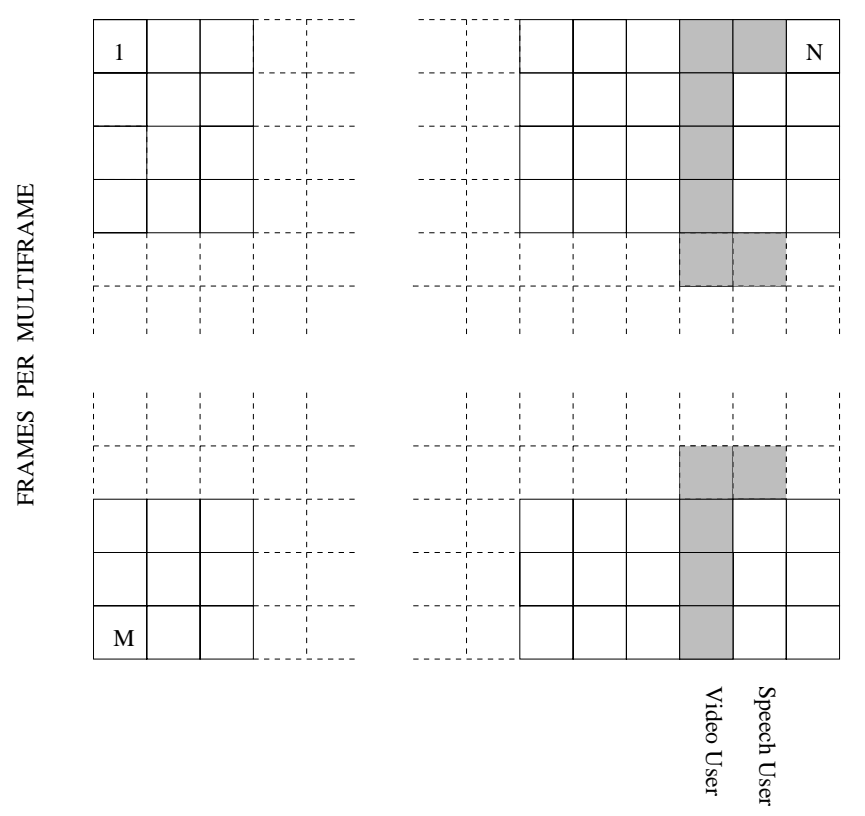

Figure 12: Multiframe comprising $M$ frames - example for slots occupied by a speech and a video user

\section{PERFORMANCE OF MF-PRMA FOR VIDEO SERVICES}

In this Section, video services of different rates are examined in the environment of the proposed MF-PRMA protocol. We investigated medium access for mean FEC-coded video target rates of $64 \mathrm{kbps}, 1 \mathrm{Mbps}$ and $3 \mathrm{Mbps}$. Video sequences were generated using the video source model described during our previous discussions. Assuming that the considered services are interactive, delay contraints of $30 \mathrm{~ms}$ for high quality video are imposed. For calculating the average delay, only the successfully arrived packets are taken into account. The delay curves must thus always be considered in conjunction with the packet dropping probability performance. In order to avoid the unrealistic situation that all terminals start contending at the same time, i.e at the beginning of the simulation, the packet generation in a terminal starts randomly in one of the first 120 frames. All simulations correspond to 260 seconds of video transmission.

Table 1 summarises the parameters for our $1 \mathrm{Mbps}$, 30ms-latency, 50 Mbps carrier-rate WLAN video service. The up- and downlink channel are accommodated in a Time Division Duplex (TDD) frame. It is assumed that 29 slots out of the total of 64 slots are available for the uplink partition. Each packet carries 424 bits of payload. FEC coding, packet header and the quasi-periodic extension of the employed Orthogonal Frequency Division Multiplex (OFDM) modulation technique require the remaining bits in each 1024-bit packet. Therefore, the previously mentioned reservation rate of one slot per frame is 424 bits $/ 1.31 \mathrm{~ms}=$ $323.66 \mathrm{Kbps}$, and the available uplink channel rate is 29 . $323.66 \mathrm{Kbps}=9.386 \mathrm{Mbps}$, where the frame duration $T_{F}$ is
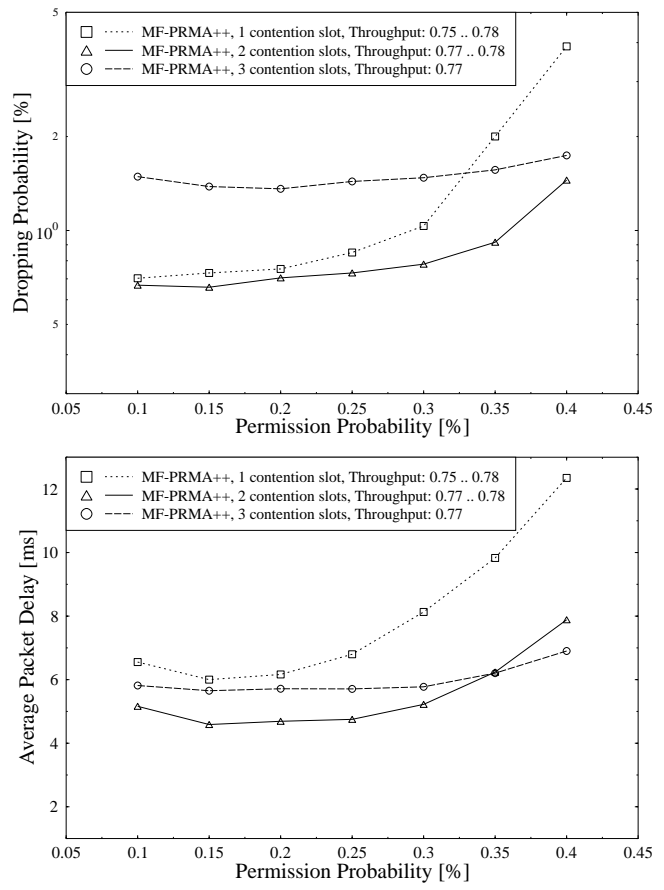

Figure 13: MF-PRMA++ simulations for $1 \mathrm{Mbps}$ video service. Examination for different numbers of contention slots.

$1.31 \mathrm{~ms}$, derived from Table 1 . We will show in the context of Figure 13 that this allows 7 video users to transmit with a packet dropping probability smaller than $0.2 \%$, if the corresponding set of parameters is taken into account. Figure 8 of Reference [16] portrays the expected video performance of an H.263-based video transceiver for various packet loss or frame error rates (FER), demonstrating that FERs as high as $5 \%$ can be tolerated by the H.263 scheme, if appropriate transmission techniques are invoked. At the previously mentioned $0.2 \%$ FER no perceived video degradation was noted and hence this value can be treated as a very conservative design target. ${ }^{2}$ Considering the MF-PRMA protocol, the target rate of $1 \mathrm{Mbps}$ is higher than the 323 kbps reservation rate of one slot per frame, requiring on average of 3-4 slots per frame. As the bitrate fluctuates, additional slots must be assigned and released sufficiently quickly and hence no reservation expiry time is imposed.

For the video simulations, three different types of PRMA protocols were examined. Namely, the proposed MF-PRMA scheme, MF-PRMA++ and MF-PRMA++ with adaptive permission probability. In MF-PRMA++, a certain number of slots per frame is used only for contentions, as in the PRMA ++ protocol. The remaining slots of the uplink partition carry non-contending packets. Apart from this, the MF-PRMA++ structure is equivalent to the MF-PRMA scheme. In an extended MF-PRMA ++ protocol, the permission probability decreases with an increasing number of reserved slots, as we will explain later.

\footnotetext{
${ }^{2}$ Various H.263 video demonstrations over a range of channel conditions and packet dropping rates can be viewed under http://www-mobile.ecs.soton.ac.uk by means of an MPEG player. Further papers on the corresponding wireless H.263 systems are also downloadable from this address.
} 

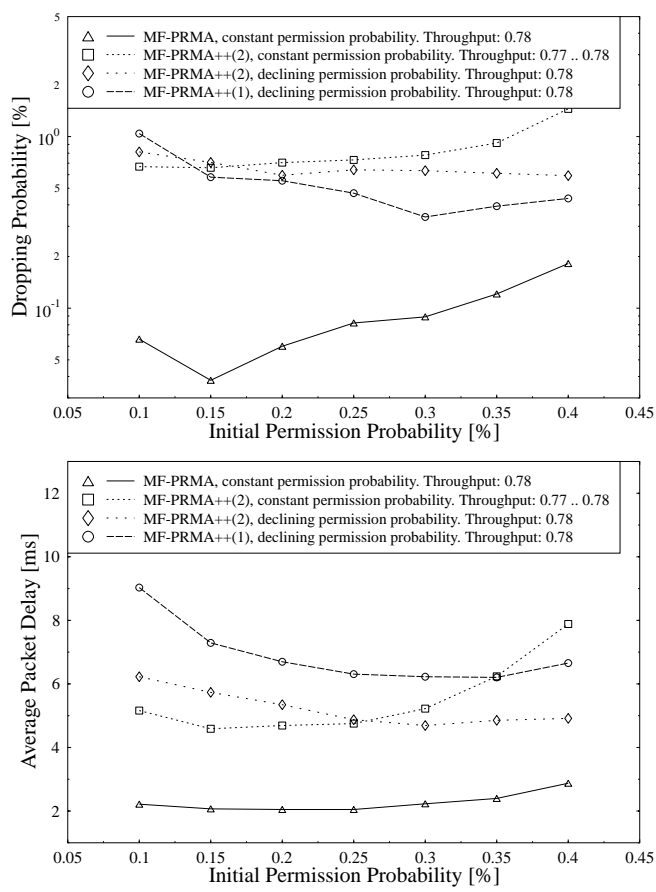

Figure 14: MF-PRMA and MF-PRMA++ simulations for $1 \mathrm{Mbps}$ video service. The figure in brackets indicates the number of contention slots.

In order to characterize MF-PRMA ++ , simulations were carried out for reserving 1,2 and 3 contention slots, considering 7 video users and an overall uplink partition of 29 slots out of a total of 64 . Figure 13 displays the associated dropping probability and average packet delay versus permission probability performances.

The system with two contention slots, that is $2 / 29 *$ $100 \%=6.9 \%$ contention bandwidth, gives the best performance for both packet dropping and average packet delay. Due to an increased number of collisions for high permission probabilities $p$ and low contention bandwidth, the delay and dropping probability performances decrease significantly for $p>0.3$ in the case of two or less contention slots. For values of $p$ between 0.1 and 0.3 , the protocol' performance is remarkably steady.

In the next series of simulations, the MF-PRMA++ performance described above will be compared to that of a MF-PRMA system. In addition, a novel variant of MFPRMA ++ is examined, which involves an adaptively decreasing permission probability for an increasing number of reserved slots. This approach, which does not require any additional signalling, is chosen due to the following observations: Initial simulations showed that although only 3-4 slots are needed on average by each terminal for the 1 Mbps video service, the system performance increased significantly if the terminal was allowed to reserve more slots when its buffer queue was much longer than that of other terminals. For this reason, each terminal was allowed to reserve up to 10 slots per frame. With acknowledgements for successful contentions only being given in the broadcast cell once a 64-slot TDD/MF-PRMA frame, however, a terminal keeps contending until the next broadcast cell arrives, even if contentions earlier in the same frame were successful and hence it does not have any more packets to transmit. In order to lessen this effect without reducing
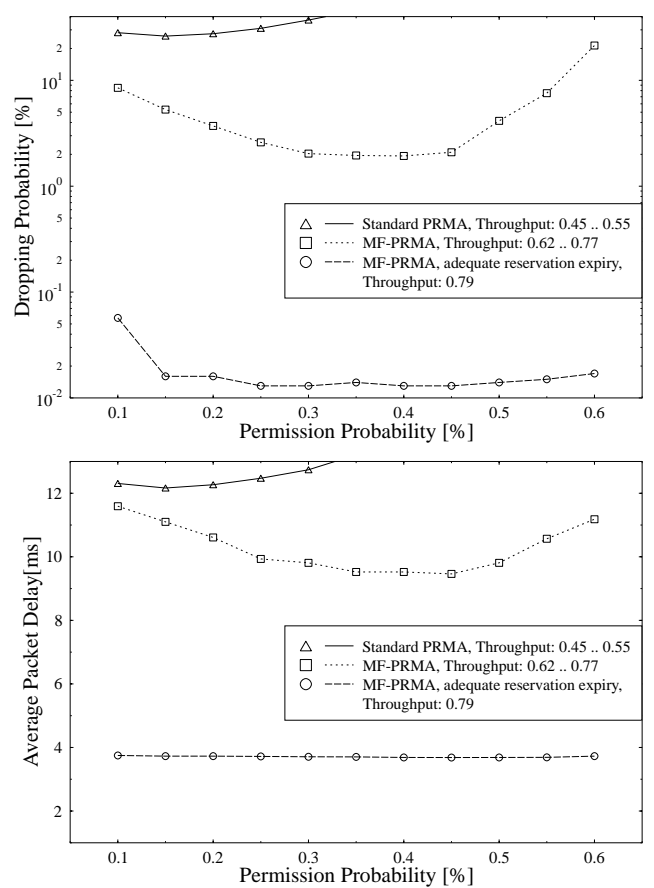

Figure 15: PRMA and MF-PRMA simulations for a $64 \mathrm{kbps}$ video service. A reservation expiry time of 1 slot is adequate in these simulations. Table 2 shows the system parameters.

the maximum number of reserved slots per frame, above we proposed a decreasing permission probability. Initial simulations showed that a slower than linear decline with an exponent of about 0.7 yielded a good performance. Results for the MF-PRMA++ approach with declining permission probability are presented in Figure 14, along with results for the MF-PRMA system without contention slots.

Explicitly, Figure 14 shows that in terms of both delay and dropping probability performance, the MF-PRMA++ approach is inferior to the MF-PRMA scheme. This was not anticipated, but it is the consequence of accupying slots for the contentions, a disadvantage, which outweighs the advantage of ensuring no contentions for traffic slots. Whereas the average packet delay for this MF-PRMA protocol is nearly independent of the permission probability in the simulated range $0.10<p<0.45$, the packet dropping probability is clearly minimized for $p=0.15$. In comparison of MF-PRMA++ and MF-PRMA++ with reducing permission probability, the latter scheme yields a slightly better performance. However, further work is needed to exploit the potential of this idea, which was also investigated in [25] using explicit signalling.

For the next set of simulations, a $64 \mathrm{kbps}$ video service, the parameters are displayed in Table 2. Again, a 30 frames-per-second video service is considered and packets are discarded after $30 \mathrm{~ms}$. Assuming a high traffic load scenario, eight video users share two columns of the multiframe, all contending for the reservation rate associated with reserving one slot every fourth frame, which corresponds to a reservation rate of $80.87 \mathrm{kbps}$. In our simulations, we compared standard PRMA, MF-PRMA and MF-PRMA with an adequate reservation expiry time. Without a reservation expiry time, the reservation is cancelled as soon as one slot is unused, which happens frequently because the source target rate, $64 \mathrm{kbps}$, is lower than the bitrate of the virtual $80.87 \mathrm{kbps}$ channel. Therefore frequent contentions 


\begin{tabular}{lccc}
\hline Definition & Notation & Unit & Value \\
\hline Channel Rate & $R_{C}$ & Mbps & 50.0 \\
Source Target Rate & $R_{S}$ & Mbps & 3.0 \\
Gross Slot Size & $S_{G}$ & bit & 1024 \\
Net Slot Size & $S_{N}$ & bit & 424 \\
Slots per Frame & $N$ & & 64 \\
Maximum Delay & $D_{\max }$ & sec. & 0.03 \\
Max. Number of Reserved & & & \\
$\quad$ Slots per Frame per Terminal & $n_{R}$ & & 15 \\
No. of Considered Upl. Slots & $N_{U}$ & & $29 . .31$ \\
Frames per Multiframe & $M$ & & 16 \\
Reservation Expiry Time & $T_{e x p}$ & slots & 0 \\
Permission Probability & $p$ & & $0.2 \ldots 0.6$ \\
Number of Video Terminals & $\mathrm{U}$ & & 3
\end{tabular}

[13] P. Cherriman, L. Hanzo: H261 and H263based Programable Video Transceivers, Proc. of ICCS'96/ISPAC'96 Singapore, Westin, 25-29. Nov. 1996, pp 1369-1373

[14] P. Cherriman, L. Hanzo: Robust H.263 Video Transmission Over Mobile Channels in Interference Limited Environments, Proc. of First Wireless Image/Video Communications Workshop, 4-5 Sept, 1996, Loughborough, UK, pp 1-7

[15] P. Cherriman, L. Hanzo: Power-controlled H.263based wireless videophone performance in interferencelimited scenarios, PIMRC'96, Taipei, Taiwan, 15-18 Oct., 1996, pp 158-162

[16] P. Cherriman, L. Hanzo: Programable H.263-based wireless video transceivers for interference-limited environments, to appear in IEEE Tr. on CSVT, 1997

[17] T. Keller, P. Cherriman, L. Hanzo: Orthogonal Frequency Division Multiplex transmission of H.263 encoded video over wireless ATM networks, to appear in Proceeding of ACTS Summit'97, Aalborg, Denmark, October, 1997

Table 3: Parameters for the 3 Mbps video service

[4] Paul Skelly, Mischa Schwartz and Sudhir Dixit. A histogram-based model for video traffic in an ATM multiplexer. IEEE/ACM Transactions on networking, Vol. 1, No. 4, pages 446-459, Aug 1993.

[5] D. Habibi, S. Gabrielsson and Z. Man. A multiplexed four layers Markov model for queueing studies of Mpeg traffic . IEEE ICCS/ISPACS '96 Singapore, pages 1180-1184, Nov 1996.

[6] J. Cosmas, G. Petit, R. Lehenert, C. Blondia, K. Kontovassilis, O. Casals, T. Theimer. A review of voice, data and video traffic models for ATM European Transactions on Telecommunications Vol. 5, No. 2, pages 139-154, Mar-Apr 1994.

[7] Daniel P. Heymann and T.V. Lakshman . Source models for VBR broadcast-video traffic. IEEE/ACM Transactions on networking Vol 4, pages 40-48, Feb 1996.

[8] Simon S. Lam, Simon Chow and David K.Y.Yau. A lossless smoothing algorithm for compressed video. IEEE/ACM Transactions on networking Vol. 4, pages 697-708, Oct 1996.

[9] ITU-T, Recommendation H.263: Video coding for low bitrate communication, 1996.

[10] M. W. Whybray and W. Ellis, "H.263 - video coding recommendation for PSTN videophone and multimedia," in IEE Colloquium (Digest), pp. 6/1-6/9, IEE, England, Jun 1995.

[11] M. Khansari, A. Jalali, E. Dubois, and P. Mermelstein, "Low bit-rate video transmission over fading channels for wireless microcellular systems," IEEE Transactions on Circuits and Systems for Video Technology, vol. 6, pp. 1-11, February 1996.

[12] N. Färber, E. Steinbach, and B. Girod, "Robust H.263 video transmission over wireless channels," in Proc. of International Picture Coding Symposium (PCS), (Melborne, Australia), pp. 575-578, March 1996.

[18] W. H. Press, S. A. Teukolsky, W. T. Vetterling and B. P. Flannery:Numerical Recipes in C. Cambridge University Press, 1994.

[19] David J. Goodman and Sherry X. Wei. Efficiency of Packet Reservation Multiple Access. IEEE Tr. on Veh. Technology, 40(1):170-176, Feb. 1991.

[20] Sanjiv Nanda, David J. Goodman and Uzi Timor. Performance of PRMA: A Packet Voice Protocol for Cellular Systems. IEEE Tr. on Veh. Technology, 40(3):584598, Aug. 1991.

[21] J. Dunlop, D. Robertson, P. Cosimi and J. De Vile: Development and Optimisation of a Statistical Multiplexing Mechanism for ATDMA, Proc. IEEE 44th Vehicular Technology Conference, Stockholm, June, 1994, pp $1040-1044$

[22] N. Amitay and S. Nanda. Resource Auction Multiple Access (RAMA) for Statistical Multiplexing of Speech in Wireless PCS. IEEE Transaction on vehicular tecnology Vol. 43 No. 3, pages 584-595, Aug 1994.

[23] V. O. K. Li and X. Qiu. Personal Communication Systems (PCS). IEEE Proceedings Vol. 83 No. 9, pages 1210-1243, Sept 1995.

[24] X. Qiu and V. O. K. Li. Dynamic Reservation Multiple Access (DRMA): A new multiple access scheme for Personal Communication System (PCS). Wireless Networks Vol. 2, pages 117-128, 1996.

[25] F. Delli Priscoli. Adaptive Parameter Computation in a PRMA, TDD Based Medium Access Control for ATM Wireless Networks. In 1996 IEEE Global Telecommunications Conference, Globecom 96, Conference Record, pages 1779-1783, Nov. 1996. 